\title{
Efeitos da Recuperação Passiva e Ativa sobre a Dor e a Funcionalidade Após Exercícios Resistidos
}

\author{
Bruno Cesar Correa Arbiza ${ }^{1}$, Natiele Camponogara Righi ${ }^{2}$, \\ Anelise Lunardi Delevati ${ }^{3}$, Léo José Rubin Neto ${ }^{4}$, Antonio Marcos Vargas da Silva ${ }^{5}$, \\ Gustavo Orione Puntel ${ }^{6}$, Luis Ulisses Signori ${ }^{7}$
}

\begin{abstract}
RESUMO
Exercícios resistidos (ER) realizados de forma intensa resultam no surgimento de dor muscular de início tardio (DMIT) e na redução da funcionalidade. Diferentes formas de recuperação após os exercícios vêm sendo estudadas para atenuar o desconforto e melhorar a funcionalidade. O objetivo do presente estudo foi avaliar os efeitos das recuperações passiva (RP) e ativa (RA) sobre a DMIT e a funcionalidade de voluntários fisicamente ativos após sessão de ER. A amostra foi composta por 15 voluntários, com idade média de $24,2( \pm 2,2)$ anos e índice de massa corporal de $24,7( \pm 2,5) \mathrm{kg} / \mathrm{m}^{2}$, submetidos ao protocolo de ER e às formas de recuperação. As sessões de ER (agachamento, cadeira extensora e leg press) consistiram em 4 séries de 10 repetições, com $80 \%$ da carga máxima (intervalo de uma semana entre a avaliação e as sessões). A DMIT foi avaliada por meio da Escala Visual Analógica 24 h, 48 h e 72 h após sessão de ER. A funcionalidade (força muscular avaliada pelo pico de torque isométrico, flexibilidade, potência, agilidade, velocidade e resistência à fadiga) foi avaliada 30 min após as intervenções (RA e RP). A RA foi mais eficiente na redução da DMIT em relação à RP nos três momentos avaliados $(24 \mathrm{~h}:-1,3$, $48 \mathrm{~h}:-1,3$ e $72 \mathrm{~h}:-1,5$ pontos; $p<0.005)$. A RA melhorou em aproximadamente $9 \%$ a força muscular dos membros inferiores em relação à RP. As demais variáveis funcionais não apresentaram diferenças entre as recuperações. A RA diminui a DMIT e atenua a perda da força muscular após ER em voluntários fisicamente ativos.
\end{abstract}

Palavras-chave: Terapia por exercício. Mialgia. Fadiga. Recuperação de função fisiológica.

\section{EFFECTS OF PASSIVE AND ACTIVE RECOVERY ABOUT PAIN AND FUNCTIONALITY AFTER RESISTED EXERCISES}

\section{ABSTRACT}

Resistance exercise (ER) performed at high intensity results in the delayed onset muscle soreness (DOMS) and diminishes functionality. Different forms of recovery after exercise have been studied to alleviate discomfort and improve functionality. The aim of the present study was to evaluate the effects of passive (PR) and active (AR) recovery on DOMS and the functionality of physically active volunteers after RE session. The sample consisted of 15 volunteers, with a mean age of $24.2( \pm 2.2)$ and body mass index of $24.7( \pm 2.5) \mathrm{kg} / \mathrm{m}^{2}$, submitted to ER protocol and the forms of recoveries. The RE sessions (squats, extension chair and leg press) consisted of 4 sets of 10 repetitions with $80 \%$ of the maximum load ( 1 week interval between evaluation and sessions). The DOMS was evaluated by Visual Analog Scale $24 \mathrm{~h}, 48 \mathrm{~h}$ and $72 \mathrm{~h}$ after RE session. Functionality (muscle strength assessed by isometric peak torque, flexibility, power, agility, speed and fatigue strength) was assessed 30 min after the interventions (AR or PR). The AR was more efficient in reducing the DOMS in relation to the PR in the three evaluated moments $(24 \mathrm{~h}:-1,3,48 \mathrm{~h}:-1,3$ and $72 \mathrm{~h}:-1,5$ points; $\mathrm{p}<0.005)$. AR improved approximately $9 \%$ of lower limb muscle strength compared to PR. The other functional variables showed no differences between recoveries. RA decreases DOMS and attenuates muscle strength loss after RE in physically active volunteers.

Keywords: Exercise therapy. Myalgia. Fatigue. Recovery of function.

RECEBIDO EM: 7/11/2019

MODIFICAÇÕES SOLICITADAS EM: 18/11/2019

ACEITO EM: 20/3/2020

Graduado em Fisioterapia pela Universidade Federal de Santa Maria (UFSM), mestrando do Programa de Pós-Graduação em Educação Física da UFSM. http://lattes.cnpq.br/4956252451266650. http://orcid.org/0000-0003-0514-0857. ftbrunocorrea@gmail.com

Graduada em Fisioterapia pela Universidade Federal de Santa Maria. Mestrado em Ciências da Reabilitação pela Universidade Federal de Santa Maria. http://lattes.cnpq.br/1029302257725197. http://orcid.org/0000-0001-7621-7204. natirighi@gmail.com

Graduando em Fisioterapia pela Universidade Federal de Santa Maria. http://lattes.cnpq.br/6104584293360005. http://orcid.org/0000-0002-4108-6459. ane-lunardi@hotmail.com

${ }^{4}$ Graduando em Fisioterapia pela Universidade Federal de Santa Maria. http://lattes.cnpq.br/4366590205469401. http://orcid.org/0000-0003-2682-238X. leojose01@hotmail.com

Graduado em Fisioterapia pela Universidade de Cruz Alta, mestre em Fisiologia pela Universidade Federal do Rio Grande do Sul e doutor em Fisiologia pela Universidade Federal do Rio Grande do Sul. http://lattes.cnpq.br/9981854873337898. http://orcid.org/0000-0001-8127-6911. antonio.77@terra.com.br

${ }^{6}$ Graduado em Fisioterapia pela Universidade Federal de Santa Maria. Mestrado e Doutorado em Ciências Biológicas (Bioquímica Toxicológica) pela Universidade Federal de Santa Maria. http://lattes.cnpq.br/0319301096075015. http://orcid.org/0000-0002-1641-9477.gustavopuntel@yahoo.com.br

Graduado em Fisioterapia pela Universidade de Cruz Alta, Mestrado em Engenharia de Produção pela Universidade Federal do Rio Grande do Sul e Doutorado e Pós-Doutorado em Ciências da Saúde no Instituto de Cardiologia do Rio Grande do Sul. http://lattes.cnpq.br/8302481057315339http://lattes.cnpq. br/8302481057315339. http://orcid.org/0000-0001-7784-9940. I.signori@hotmail.com 


\section{INTRODUÇÃO}

Diversos programas de exercícios resistidos (ER), usualmente realizados nas academias de musculação, são amplamente utilizados para manter e/ ou melhorar a funcionalidade (KANG; KIM; LEE, 2018; KELLMANN et al., 2018), sendo empregados na reabilitação (HISLOP et al., 2019) e no treinamento físico (MOREL; HAUTIER, 2017). Quando, entretanto, realizados em alta intensidade e de forma aguda, a maior produção de espécies reativas de oxigênio e nitrogênio pode exceder a capacidade antioxidante, induzindo um estado transitório de estresse oxidativo (POWERS; JACKSON, 2008) e a maior concentração de marcadores inflamatórios (TEIXEIRA et al., 2014a), provocando dano muscular, o que diminui a funcionalidade e o desempenho musculoesquelético (DABBS; CHANDER, 2018; MIKA et al., 2007, 2016). Por outro lado, a médio e a longo prazos, a inflamação, advinda desses exercícios, media o processo de reparo e promove a regeneração, o que faz parte do processo normal de remodelamento muscular advindo do treinamento (CALLE; FERNANDEZ, 2010).

Clinicamente, a resposta inflamatória manifesta-se pela dor muscular de início tardio (DMIT) e pela redução da capacidade de realizar diferentes variáveis funcionais (CROWTHER et al., 2019; KARGARFARD et al., 2016). A DMIT tem início entre 6 a 24 horas (h), com pico entre $48 \mathrm{~h}$ a $72 \mathrm{~h}$ e desaparece de 5 a 7 dias após os exercícios (KANG; KIM; LEE, 2018). Esse sintoma é mais evidente em indivíduos destreinados (MISSAU et al., 2018), em esportistas iniciantes e em reabilitação (ORTIZ et al., 2018), mas também afeta atletas de alto rendimento (KELLMANN et al., 2018; ORTIZ et al., 2018). A redução da funcionalidade após ER caracteriza-se pela diminuição da força (KANG; KIM; LEE, 2018; MIKA et al., 2016; TAIPALE et al., 2018), da flexibilidade (CHARLTON et al., 2018), da agilidade (KARGARFARD et al., 2016) e da potência (FERRARI et al., 2013) e pode predispor ao aparecimento de lesões (KELLMANN et al., 2018; POL et al., 2018; SOLIGARD et al., 2016).

$\mathrm{Na}$ tentativa de atenuar esses efeitos, diferentes estratégias não farmacológicas vêm sendo estudadas (CROWTHER et al., 2019; DUPUY et al., 2018; ORTIZ et al., 2018). Dentre as diferentes formas de recuperação, a mais utilizada pela população em geral é a recuperação passiva (RP) por meio de repouso (KARGARFARD et al., 2016; MISSAU et al., 2018; MOREL; HAUTIER, 2017). A RP normalmente é utilizada como a recuperação controle em pesquisas científicas. Em oposição a essa, os exercícios aeróbicos de baixa intensidade são considerados uma forma ativa de recuperação após os exercícios físicos.

Ao nosso conhecimento, até o presente momento nenhum estudo foi conduzido para comparar a efetividade destas estratégias de recuperação em relação à DMIT e às variáveis funcionais, que envolvam a amplitude de movimento (ADM), potência, agilidade, velocidade e resistência à fadiga de homens fisicamente ativos após ER. Além disso, a eficácia da RA permanece incerta em relação às variáveis da função musculoesquelética (VAN HOOREN; PEAKE, 2018). Nesse sentido, o objetivo deste estudo foi comparar os efeitos da RP e da RA sobre a DMIT e a funcionalidade de voluntários fisicamente ativos após sessão de ER.

\section{MATERIAIS E MÉTODOS}

\section{Aspectos éticos}

A presente pesquisa (projeto: $n$ ㅇ 047670) foi aprovada pelo Comitê de Ética em Pesquisa na Área da Saúde (protocolo: 2.538.028) da Universidade Federal de Santa Maria (UFSM). A mesma respeita a Declaração de Helsinque (2013) e a Resolução 466/2012 do Conselho Nacional de Saúde para pesquisas com seres humanos. Todos os voluntários foram instruídos sobre os objetivos da pesquisa e assinaram o Termo de Consentimento Livre e Esclarecido.

\section{Critérios de elegibilidade}

Os critérios de inclusão compreenderam voluntários do sexo masculino, alfabetizados, com idade entre 18 e 35 anos, fisicamente ativos (IPAQ (HAGSTRÖMER; OJA; SJÖSTRÖM, 2006)), com o índice de massa corporal (IMC) entre 20 e $30 \mathrm{~kg} / \mathrm{m}^{2}$, não fumantes, que não estivessem em uso de suplementos alimentares ou de medicamentos. Os voluntários que apresentaram sintomas de distúrbios musculoesqueléticos, diagnóstico prévio de doenças reumatológicas, cardiovasculares, neurológicas, oncológicas, imunológicas, hematológicas e problemas psiquiátricos e/ou cognitivos e que consumiam bebidas alcoólicas (mais de uma vez na semana), não foram incluídos. Nos dias das avaliações e intervenções os voluntários que apresentassem sintomas de distúrbios musculoesqueléticos ou que tivessem realizado exercício físico intenso nas últimas 48 horas, seriam excluídos da pesquisa.

\section{Cálculo amostral}

O cálculo amostral foi baseado em estudo anterior (MISSAU et al., 2018) e estimou-se que seriam necessários 15 voluntários para a realização deste. 
Após a realização de sessão de ER igual a do presente estudo e aplicação da imersão da água fria, o cálculo apresentou uma diferença na DMIT 24h após ER de 2,3 pontos (Escala Visual Analógica -EVA) entre as sessões (recuperação passiva vs recuperação com imersão na água fria) e desvio padrão de 2,6 pontos. Esses valores foram mantidos para um poder de $90 \%$ e $\alpha=0,05$.

\section{Desenho do estudo e procedimentos}

Inicialmente foram avaliados os critérios de elegibilidade e executadas as adaptações às avaliações funcionais e ao protocolo de ER. A partir deste encontro, o estudo foi feito em mais três momentos, com sete dias de intervalo entre eles. No primeiro momento foram realizadas as medidas de variáveis biométricas (idade, peso, altura), avaliações funcionais (Basal) e da força muscular (Basal, avaliada pelo teste de 10 Repetições Máximas). No segundo e terceiro momentos, foram realizados o protocolo de $E R$, as intervenções (RP e RA) e a avaliação das variáveis funcionais 30 minutos após.

As avaliações e as intervenções foram feitas em salas separadas. As recuperações e a sequência dos ER foram randomizadas pelo software www.random.org, e a alocação foi guardada em envelope pardo (aberta somente no segundo e terceiro momentos). Os avaliadores foram mantidos cegos às intervenções.

\section{Avaliação e prescrição dos exercícios resistidos}

O teste de dez repetições máximas (10RM) foi utilizado para avaliar a força muscular e, baseado nesta, prescrever os ERs. Antes de iniciar o teste, os sujeitos foram submetidos ao aquecimento (sem carga) específico para cada exercício (agachamento, cadeira extensora e leg press). Os valores de carga de trabaIho foram atingidos entre três e cinco tentativas (FOSTER et al., 2001). A manipulação da carga foi realizada usando $5 \mathrm{~kg}$ em cada nova tentativa, com um intervalo de 2 a 3 minutos entre cada série e 4 minutos entre os exercícios (BRITO et al., 2020; MISSAU et al., 2018; TEIXEIRA et al., 2014a, 2014b). Os voluntários receberam estímulos verbais durante as avaliações. A maior carga suportada (falha muscular concêntrica) foi de $100 \%$ das 10RMs para cada ER selecionado.

A sessão de ER foi composta por três exercícios (cadeira extensora, agachamento livre e leg press). Antes da realização dos exercícios, os sujeitos foram submetidos a um aquecimento específico em cada exercício (1 série de 15 repetições com $40 \%$ da carga máxima obtida no teste de 10RMs). As sessões de exercícios foram compostas por 4 séries de $80 \%$ de
10RMs, com intervalos de 1 minuto entre as séries e 2 minutos entre os exercícios (BRITO et al., 2020; MISSAU et al., 2018; TEIXEIRA et al., 2014a, 2014b).

\section{Intervenções}

Durante a RP os voluntários permaneceram 20 minutos em repouso, sentados sobre o cicloergômetro, semelhante ao realizado em estudos prévios com modificações (MISSAU et al., 2018; TEIXEIRA et al., 2014a). A RA consistiu em pedalar no cicloergômetro (LX130, Movement, São Paulo, Brasil) por 20 minutos, com intensidade entre 50 a 60 RPMs, de forma contínua (MIKA et al., 2016). A carga de $9 \pm 1 \mathrm{~W}$ foi ajustada individualmente para se alcançar $50 \%$ da frequência cardíaca máxima teórica (100 BPM) (VANDERTHOMMED; MAKROF; DEMOULIN, 2010).

Ambas as intervenções foram realizadas em sala fechada, silenciosa e climatizada $\left(24 \pm 1^{\circ} \mathrm{C}\right)$. Os voluntários receberam água antes dos exercícios e lhes foi restringida a ingestão desta durante a realização das sessões de exercícios.

\section{Desfechos}

O desfecho primário foi a DMIT e os desfechos secundários compreenderam as variáveis funcionais (força, flexibilidade, potência, agilidade, velocidade e resistência à fadiga).

\section{Avaliação dos desfechos}

A DMIT foi avaliada 24 h, 48 h e 72 h após o protocolo de ER por meio da EVA enviada por mensagem via WhatsApp, solicitando a realização de um único agachamento sem carga (KARGARFARD et al., 2016).

A força muscular foi avaliada pelo pico de torque isométrico de quadríceps, por meio de um dinamômetro de tração-compressão (EMG System, São José dos Campos, Brasil), com capacidade de 500 kgf e resolução de $0,1 \mathrm{~kg}$, um conjunto de cabos de fixação e uma placa conversora A/D (EMG System, São José dos Campos, Brasil). O voluntário permaneceu sentado, com apoio para as costas, em postura ereta e 90 응 de flexão de quadril. 0 ângulo para avaliação do pico de torque isométrico de extensores de joelho foi em 60 어 de flexão do mesmo (DABBS; CHANDER, 2018).

$\mathrm{Na}$ sequência foram realizadas as avaliações da flexibilidade [(Lunge Test (CHISHOLM et al., 2012) e banco de sentar e alcançar de Wells e Dillon (SIGNORI et al., 2008)], da potência (Single Hop Test (BOLGLA; KESKULA, 1997)), da agilidade [ $T$ Test (LATORRE ROMÁN; VILLAR MACIAS; GARCÍA PINILLOS, 2017)], 
da velocidade (Teste de 30 metros (PEARCEY et al., 2015)) e da resistência à fadiga (Teste Senta e Levanta (TVETER et al., 2014)). A sequência das avaliações foi mantida em todos os momentos. $O$ intervalo de $2 \mathrm{mi}-$ nutos foi adotado entre cada protocolo de avaliação.

\section{Análises estatísticas}

Os dados estão apresentados em média e desvio padrão ( \pm DP). O teste de Shapiro-Wilk foi utilizado para verificar a distribuição dos dados. As comparações entre as intervenções foram realizadas pela Anova de uma e duas vias para medidas repetidas, ambas seguidas pelo post hoc de Newman-Keuls. Os resultados significantes estão apresentados na forma de diferenças médias (DM) e os seus respectivos intervalos de confiança de $95 \%$ (IC 95\%). A taxa de erro $\alpha$, de $5 \%$ $(p<0,05)$, foi considerada significante.

\section{RESULTADOS}

A amostra inicial compreendeu 22 voluntários que foram recrutados para esta pesquisa. Dois foram excluídos, pois apresentavam sintomas ou dores musculoesqueléticas no dia do exame. Vinte voluntários realizaram as avaliações basais, sendo cinco excluídos das análises por falta de seguimento (três não compareceram na RP e dois na RA). O fluxograma do estudo encontra-se na Figura 1. A amostra compreendeu 15 voluntários do sexo masculino, com idade média de $24,2 \pm 2,2$ anos e IMC de $24,7 \pm 2,5 \mathrm{~kg} / \mathrm{m}^{2}$. Destes, 12 (86\%) apresentavam dominância no membro inferior direito. Nenhum voluntário relatou qualquer efeito adverso, além da já esperada DMIT advinda do protocolo de ER.

Figura 1 - Fluxograma do Estudo

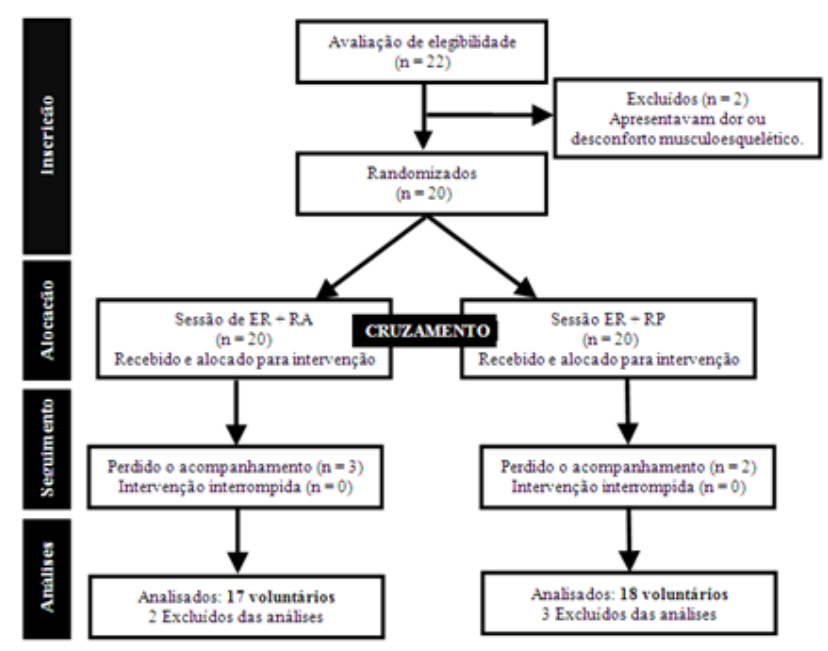

n = número. ER: Exercícios Resistidos; RA: Recuperação Ativa; RP: Recuperação Passiva.

Fonte: Dados da pesquisa.
Os resultados da DMIT ( $24 \mathrm{~h}, 48 \mathrm{~h}$ e 72 h) estão apresentados na Figura 2. Os voluntários não apresentaram dor (0 pontos) antes das sessões de ER. Conforme esperado, os valores aumentaram $24 \mathrm{~h}$ (RP: $5,0 \pm$ 1,3 pontos; RA: $3,9 \pm 1,6$ pontos), 48 h (RP: $3,4 \pm 1,4$ pontos; RA: $2,2 \pm 1,5$ pontos) e $72 \mathrm{~h}$ (RP: $2,4 \pm 2,0$ pontos; RA: $0,8 \pm 0,9$ pontos) após as sessões de ER.

Figura 2 - Resultados da dor muscular de início tardio (DMIT) avaliados $24 \mathrm{~h}, 48 \mathrm{~h}$ e $72 \mathrm{~h}$ após sessão de exercícios resistidos

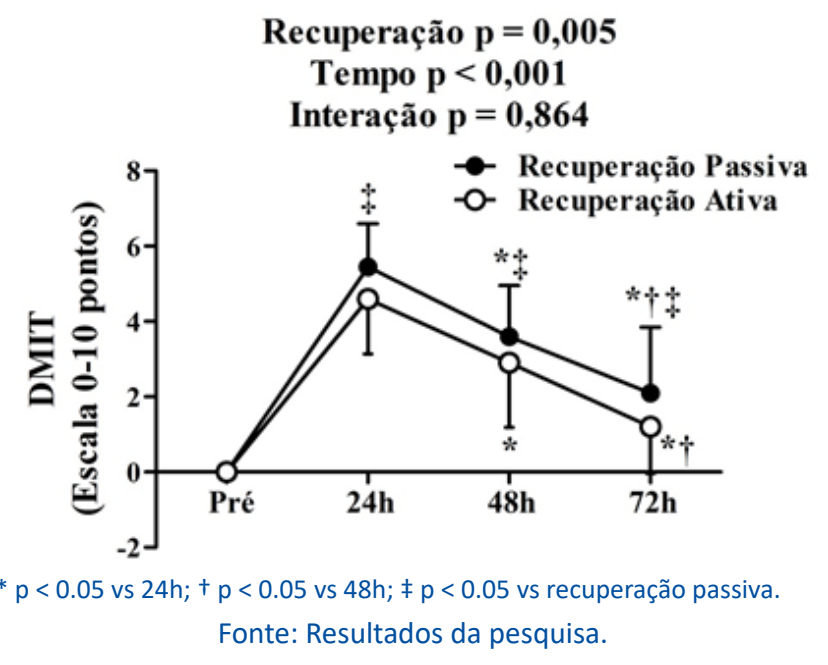

Após $24 \mathrm{~h}$, as recuperações (RP e RA) apresentaram redução da DMIT ao longo do tempo $(p<0.001)$. $\mathrm{Na} R P$, em comparação a $24 \mathrm{~h}$ após $E R$, a redução $(p=$ 0.001 ) foi de $34,2 \%$ em 48 h (DM: -1,88; IC 95\%: -1.1 a $-2,6$ pontos) e de $58,5 \%$ em 72 h (DM: $-3,2$; IC $95 \%$ : -2.5 a $-3,9$ pontos); e entre 48 h e 72 h esta redução foi de 37\% (DM: -1,3; IC 95\%: -0,6 a -2,1). Na RA, em comparação a $24 \mathrm{~h}$ após $E R$, a redução $(p=0,001)$ foi de $43,6 \%$ em 48 h (DM: -1,8; IC 95\%: -1.1 a $-2,5$ pontos) e de $80,6 \%$ em 72 h (DM: -3,3; IC 95\%: $-2,6$ a $-4,1$ ); e entre 48 h e 72 h esta redução foi de $65,7 \%$ (DM: -1,3; IC 95\%: -0,6 a -2,1). Ainda, a RA apresentou melhores efeitos quando comparada à $\mathrm{RP}(p=0,005)$, pois a DMIT foi 25,4\% menor que a RP $24 \mathrm{~h}$ (DM: -1,3; IC 95\%: $-0,1$ a -2,6), 35,2\% em 48 h (DM: -1,3; IC 95\%: -0.04 a $-2,5)$ e $70 \%$ após 72 h (DM: -1,5; IC 95\%: -0,2 a -2,7).

Os resultados da avaliação do pico de torque isométrico de quadríceps estão apresentados na Tabela 1 (valores absolutos). Em relação às avaliações basais, a RP apresentou uma redução de $16 \%$ no membro inferior dominante (MD) (DM: -16,2; IC 95\%: -21,1 a -11 ,2) (Figura $3 A$ ) e de $18 \%$ no membro inferior não dominante (MND) (DM: -18,5; IC 95\%: -22,9 a -14,2) (Figura 3B). Na RA, no MD essa redução foi de 7\% (DM: $-7,3$; IC 95\%: -13,2 a -1,4) e no MND de 10\% (DM: -9,6; IC 95\%: -16,1 a -3,2) em relação às avaliações basais. 
Tabela 1 - Dados das variáveis funcionais

\begin{tabular}{lcccc}
\hline \multicolumn{1}{c}{ Variáveis } & Basal & RP & RA & Valor $\boldsymbol{p}$ \\
\hline Força - PTI (kgf) & & & & \\
MD & $72,8 \pm 12,8$ & $61,2 \pm 12,9^{*}$ & $67,2 \pm 10,0^{*+}$ & $<0,001$ \\
MND & $71,2 \pm 9,9$ & $58,3 \pm 11,6^{*}$ & $64,6 \pm 9,9^{*}+$ & $<0,001$ \\
ADM - LT (cm) & & & & \\
MD & $13,1 \pm 2,3$ & $11,5 \pm 3,7$ & $12,8 \pm 2,2$ & 0,168 \\
MND & $13,1 \pm 2,2$ & $11,7 \pm 3,8$ & $12,9 \pm 2,5$ & 0,208 \\
Flexibilidade - BW (cm) & $28,7 \pm 9,4$ & $27,3 \pm 10,7$ & $29,3 \pm 9,1$ & 0,735 \\
Potência - SHT (cm) & & & & \\
MD & $131,3 \pm 18,9$ & $138,8 \pm 20,9$ & $139,3 \pm 20,6$ & 0,142 \\
MND & $130,0 \pm 19,8$ & $135,6 \pm 22,6$ & $137,7 \pm 23,0$ & 0,201 \\
Agilidade - TT (s) & $13,5 \pm 1,8$ & $13,3 \pm 1,0$ & $12,8 \pm 0,5$ & 0,257 \\
Velocidade - T30 (s) & $4,6 \pm 0,5$ & $4,6 \pm 0,2$ & $4,5 \pm 0,2$ & 0,377 \\
Resistência TSL (rep) & $17,5 \pm 2,9$ & $18,4 \pm 5,6$ & $19,9 \pm 2,2$ & 0,121 \\
\hline
\end{tabular}

Dados apresentados em média \pm desvio padrão (DP); PTI: pico de torque isométrico; MD: membro dominante; MND: membro não dominante; ADM: amplitude de movimento; LT: lunge test; cm: centímetros; BW: Banco de Welles; SHT: Single Hop Test; TT: $T$ test; T30: teste de 30 metros; TSL: teste de sentar e levantar.

$* p<0,05$ vs Basal; $+p<0,05$ vs Recuperação Passiva.

As reduções no pico de torque isométrico do quadríceps na RA (MD vs MND; $p=0,046$ ) e RP (MD vs MND; $p=0,074$ ) foram semelhantes em relação à dominância dos membros inferiores. $O$ pico de torque isométrico de quadríceps após a RA foi aproximadamente 9\% maior que após a RP para o MD (DM: -8,9; IC 95\%: $-14,8$ a -2,9) e MND (DM: -8,9; IC 95\%: -16,7 a -1,1) após sessão de ER. A Figura 3 apresenta os valores relativos (\%) do pico de torque isométrico de quadríceps.

Figura 3 - Resultados do pico de torque isométrico do quadríceps (\%)
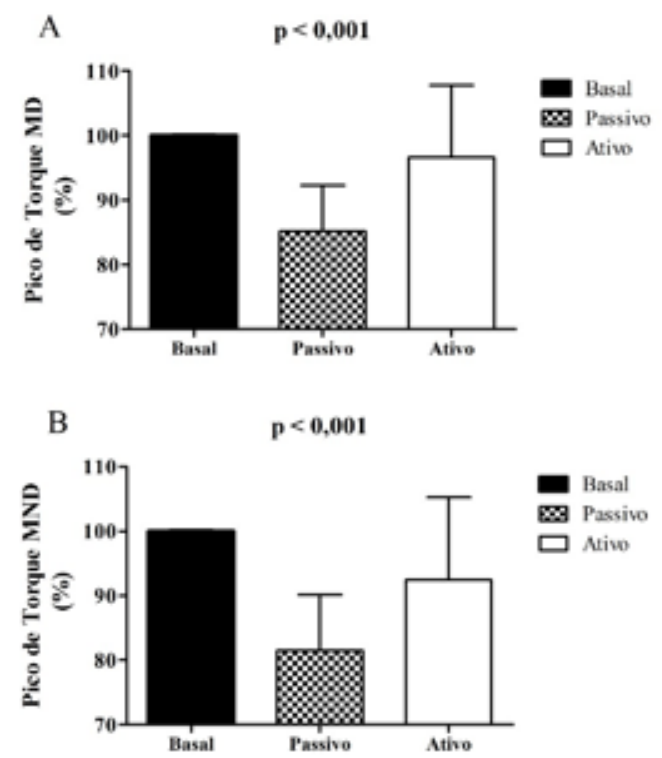

MD: membro dominante; MND: membro não dominante.

$* p<0.05$ vs basal; $+p<0.05$ vs recuperação passiva.
A Tabela 1 apresenta os resultados das demais variáveis funcionais. A amplitude de movimento articular, a flexibilidade, a potência, a agilidade, a velocidade e a resistência à fadiga não apresentaram alterações em relação às medidas basais e entre as intervenções.

\section{DISCUSSÃO}

Os principais resultados desta pesquisa demonstram que os ERs induzem a DMIT e reduzem a força muscular de quadríceps. A RA foi mais eficiente que a RP na redução da DMIT em $24 \mathrm{~h}(25,4 \%), 48 \mathrm{~h}$ $(35,2 \%)$ e 72 h (70\%) após os ERs e aumentou em $9 \%$ o pico de torque isométrico de quadríceps.

$O$ presente protocolo de ER induziu a DMIT, assim como ocorreu em estudos prévios (KANG; KIM; LEE, 2018; MISSAU et al., 2018; TEIXEIRA et al., 2014a). No presente estudo a RA foi mais eficiente na redução da DMIT que a RP. Isso, porém, não aconteceu em estudo anterior (VANDERTHOMMED; MAKROF; DEMOULIN, 2010). A controvérsia dos resultados pode estar relacionada aos diferentes tipos de exercícios (isométrico) e à carga utilizada (3 sets de 25 contrações submáximas - 60 a $55 \%$ da RM). Corroborando os resultados do presente estudo, entretanto, a recente revisão sistemática demonstrou que a RA reduz a DMIT (DUPUY et al., 2018). As alterações mecânicas e metabólicas induzem a um estado transitório de estresse oxidativo (POWERS; JACKSON, 2008) e são responsáveis pelo dano muscular e a consequente resposta inflamatória induzidos pelos 
exercícios (PEAKE et al., 2017; TEIXEIRA et al., 2014a). Após uma sessão de ER realizada em alta intensidade, ocorre o aumento de marcadores inflamatórios, da concentração plasmática de proteínas inflamatórias de fase aguda e acúmulo de ácido láctico (BRITO et al., 2020; MISSAU et al., 2018; TEIXEIRA et al., 2014a). Esse processo, em especial o ácido láctico, leva à excitação dos receptores nociceptivos e à consequente DMIT (PEAKE et al., 2017). A redução da dor após a RA está associada ao aumento do fluxo sanguíneo, pois as contrações musculares melhoram o suprimento de oxigênio e promovem a remoção dessas proteínas e dos metabólitos, favorecendo a depuração de lactato sanguíneo, resultando na diminuição da estimulação dos receptores nociceptivos (GUPTO et al., 1996).

Estudos prévios demonstram a redução da força muscular de quadríceps após ER (DABBS; CHANDER, 2018; KANG; KIM; LEE, 2018; MOREL; HAUTIER, 2017) e a eficácia da RA em atenuar tal redução (MOREL; HAUTIER, 2017). Esses resultados corroboraram os resultados desta pesquisa. Mika et al. (2016) compararam os efeitos da RA e da RP em canoístas e jogadores de futebol sobre o pico de torque, trabalho e potência muscular após uma exaustiva corrida em esteira, e observaram que a RA (realizada por 20 minutos) é mais eficiente que a RP, especialmente quando realizada nos músculos que estavam submetidos à atividade esportiva (canoístas para membros superiores e futebolistas para membros inferiores). Esses resultados reforçam que, após a aplicação de um protocolo de ER de alta intensidade para os membros inferiores (músculo quadríceps), a RA realizada em cicloergômetro por 20 minutos é eficiente.

No presente estudo a amplitude de movimento articular, a flexibilidade, a potência, a agilidade, a velocidade e a resistência à fadiga não se modificaram ante o protocolo de ER e as recuperações. Estudo prévio observou redução na agilidade, na potência vertical e no pico de torque isométrico após a realização de um protocolo de fadiga (75\% de 1RM no leg press e no agachamento) (KARGARFARD et al., 2016). Em estudo que simulou um torneio de rugby com repetidas sessões de exercícios, o desempenho (potência vertical e agilidade) e a flexibilidade foram reduzidos após o procolo de fadiga, e a RA não foi capaz de atenuar essas alterações (CROWTHER et al., 2019). As divergências entre esses resultados funcionais e o presente estudo devem-se à quantidade de variáveis envolvidas na prescrição das recuperações ativas, nos diferentes tipos de exercícios e nas diversas populações avaliadas (ORTIZ et al., 2018).
A ausência da avaliação de marcadores plasmáticos de dano muscular e inflamação é uma limitação desta pesquisa, pois limita a elucidação dos mecanismos fisiológicos envolvidos nos efeitos destas intervenções.

\section{CONCLUSÃO}

Os ERs realizados agudamente e em alta intensidade induzem a DMIT e diminuem o pico de torque isométrico. Comparada à RP, a RA reduziu a DMIT 24 h, 48 h e 72 h após sessão de ER e atenuou a diminuição da força muscular 30 min após os exercícios. A amplitude de movimento articular, a flexibilidade, a velocidade, a agilidade, a potência e a resistência à fadiga apresentaram comportamentos semelhantes ante as recuperações estudadas. Os resultados desta pesquisa demonstram que a RA favorece a recuperação da DMIT e da força muscular após os ERs.

\section{REFERÊNCIAS}

BOLGLA, L. A.; KESKULA, D. R. Reliability of lower extremity functional performance tests. The Journal of Orthopaedic and Sports Physical Therapy, v. 26, n. 3, p. 138-142, 1997.

BRITO, E. de et al. Vitamins C and E Associated With Cryotherapy in the Recovery of the Inflammatory Response After Resistance Exercise : A Randomized Clinical Trial. The Journal of Strengh and Conditioning Research, v. Im Press, n. 1, 2020.

CALLE, M. C.; FERNANDEZ, M. L. Effects of resistance training on the inflammatory response. Nutrition Research and Practice, v. 4, n. 4, p. 259-269, 2010.

CHARLTON, P. C. et al. Knee flexion strength is significantly reduced following competition in semi-professional Australian Rules football athletes: Implications for injury prevention programs. Physical Therapy in Sport, v. 31, p. 9-14, maio 2018.

CHISHOLM, M. D. et al. Reliability and validity of a weight-bearing measure of ankle dorsiflexion range of motion. Physiotherapy Canada, v. 64, n. 4, p. 347-355, 2012.

CROWTHER, F. A. et al. Effects of Various Recovery Strategies on Repeated Bouts of Simulated Intermittent Activity. Journal of Strength and Conditioning Research, v. 33, n. 7, p. 1.781-1.794, jul. 2019.

DABBS, N. C.; CHANDER, H. The Effects of Exercise Induced Muscle Damage on Knee Joint Torque and Balance Performance. Sports, Basel, Switzerland, v. 6, n. 3, set. 2018.

DUPUY, O. et al. An Evidence-Based Approach for Choosing Post-exercise Recovery Techniques to Reduce Markers of Muscle Damage, Soreness, Fatigue, and Inflammation: A Systematic Review With Meta-Analysis. Frontiers in Physiology, v. 9, p. 403, abr. 2018. 
FERRARI, H. G. et al. Efeito de diferentes métodos de recuperação sobre a remoção de lactato e desempenho anaeróbio de futebolistas. Revista Brasileira de Medicina do Esporte, v. 19, n. 6, p. 423-426, 2013.

FOSTER, C. et al. A New Approach to Monitoring Exercise Training. Journal of Strength and Conditioning Research, v. 15, n. 1, p. 109-115, 2001.

GUPTO, S. et al. Comparative Study of Lactate Removal in Short Term Massage of Extremities, Active Recovery and a Passive Recovery Period After Supramaximal Exercise Sessions. International Journal of Sports Medicine, v. 17, p. 106-110, 1996.

HAGSTRÖMER, M.; OJA, P.; SJÖSTRÖM, M. The International Physical Activity Questionnaire (IPAQ): a study of concurrent and construct validity. Public Health Nutrition, v. 9, n. 6, p. 755-762, 2006.

HISLOP, A. C. et al. Does adding hip exercises to quadriceps exercises result in superior outcomes in pain, function and quality of life for people with knee osteoarthritis? A systematic review and meta-analysis. British Journal of Sports Medicine, v. 1, n. 0, p. 1-10, fev. 2019.

KANG, M.-S.; KIM, J.; LEE, J. Effect of different muscle contraction interventions using an isokinetic dynamometer on muscle recovery following muscle injury. Journal of Exercise Rehabilitation, v. 14, n. 6, p. 1.080-1.084, 2018.

KARGARFARD, M. et al. Efficacy of massage on muscle soreness, perceived recovery, physiological restoration and physical performance in male bodybuilders. Journal of Sports Sciences, v. 34, n. 10, p. 959-965, maio 2016.

KELLMANN, M. et al. Recovery and Performance in Sport: Consensus Statement. International Journal of Sports Physiology and Performance, v. 13, n. 2, p. 240-245, fev. 2018.

LATORRE ROMÁN, P. Á.; VILLAR MACIAS, F. J.; GARCÍA PINILLOS, F. Effects of a contrast training programme on jumping, sprinting and agility performance of prepubertal basketball players. Journal of Sports Sciences, v. 21, n. 0, p. 1-7, 2017.

MIKA, A. et al. Comparison of Recovery Strategies on Muscle Performance After Fatiguing Exercise. American Journal of Physical Medicine \& Rehabilitation, v. 86, n. 6, p. 474481, jun. 2007.

MIKA, A. et al. Comparison of Two Different Modes of Active Recovery on Muscles Performance after Fatiguing Exercise in Mountain Canoeist and Football Players. PloS one, v. 11, n. 10, p. e0164216, 2016.

MISSAU, E. et al. Cold Water Immersion and Inflammatory Response After Resistance Exercises. Revista Brasileira de Medicina do Esporte, v. 24, n. 5, p. 357-361, 2018.

MOREL, B.; HAUTIER, C. A. The neuromuscular fatigue induced by repeated scrums generates instability that can be limited by appropriate recovery. Scandinavian Journal of Medicine \& Science in Sports, v. 27, n. 2, p. 209-216, fev. 2017.
ORTIZ, R. O. et al. A Systematic Review on the Effectiveness of Active Recovery Interventions on Athletic Performance of Professional-, Collegiate-, and Competitive-Level Adult Athletes. Journal of Strength and Conditioning Research, p. 1, maio 2018.

PEAKE, J. M. et al. Recovery from Exercise Muscle damage and inflammation during recovery from exercise. Journal of Applied Physiology, v. 122, p. 559-570, 2017.

PEARCEY, G. E. et al. Foam Rolling for Delayed-Onset Muscle Soreness and Recovery of Dynamic Performance Measures. Journal of Athletic Training, v. 50, n. 1, p. 5-13, 2015.

$\mathrm{POL}, \mathrm{R}$. et al. From microscopic to macroscopic sports injuries. Applying the complex dynamic systems approach to sports medicine: A narrative review. British Journal of Sports Medicine, v. 1, n. 0, p. 1-8, abr. 2018.

POWERS, S. K.; JACKSON, M. J. Exercise-induced oxidative stress: cellular mechanisms and impact on muscle force production. Physiological Reviews, v. 88, n. 4, p. 1.2431.276, 2008.

SIGNORI, L. U. et al. Efeito de agentes térmicos aplicados previamente a um programa de alongamentos na flexibilidade dos músculos isquiotibiais encurtados. Revista Brasileira de Medicina do Esporte, v. 14, n. 4, p. 328-331, 2008.

SOLIGARD, T. et al. How much is too much? (Part 1) International Olympic Committee consensus statement on load in sport and risk of injury. British Journal of Sports Medicine, $\mathrm{v}$. 50, n. 17, p. 1.030-1.041, set. 2016.

TAIPALE, R. S. et al. Active and passive recovery influence responses of luteinizing hormone and testosterone to a fatiguing strength loading. European Journal of Applied Physiology, v. 118, n. 1, p. 123-131, jan. 2018.

TEIXEIRA, A. D. O. et al. Inflammatory response after session of resistance exercises in untrained volunteers. Acta Scientiarum, v. 37, n. 1, p. 31-39, $2014 a$.

TEIXEIRA, A. D. O. et al. The Importance of Adjustments for Changes in Plasma Volume in the Interpretation of Hematological and Inflammatory Responses after Resistance Exercise. Journal of Exercise Physiology, v. 17, n. 4, p. 72-83, 2014b.

TVETER, A. T. et al. Health-Related Physical Fitness Measures: Reference Values and Reference Equations for Use in Clinical Practice. Archives of Physical Medicine and Rehabilitation, v. 95, n. 7, p. 1.366-1.373, jul. 2014.

VAN HOOREN, B.; PEAKE, J. M. Do We Need a Cool-Down After Exercise? A Narrative Review of the Psychophysiological Effects and the Effects on Performance, Injuries and the Long-Term Adaptive Response. Sports Medicine, Auckland, N.Z., v. 48, n. 7, p. 1.575-1.595, 2018.

VANDERTHOMMED, M.; MAKROF, S.; DEMOULIN, C. Comparison of active and electrostimated recovery strategies after fatiguing exerice. Journal of Sports Science and Medicine, v. 9, p. 164-169, 2010. 